\title{
Siloxane based Organic-Inorganic Hybrid Polymers and their Applications for Nanostructured Optical/Photonic Components
}

\author{
Rahmat Hidayat $^{1}{ }^{*}$, Widiyanta Gomulya ${ }^{1}$, Pina Pitriana ${ }^{1}$, Ryan Irmansyah ${ }^{1}$, \\ Rany Miranti ${ }^{1}$, Herman $^{1}$, Sahrul Hidayat ${ }^{2}$, Fitrilawati $^{2}$, Akihiko Fujii $^{3} \&$ \\ Masanori Ozaki ${ }^{3}$ \\ ${ }^{1}$ Physics of Photonics and Magnetism Research Division, Faculty of Mathematics and \\ Natural Sciences, Bandung Institute of Technology, Indonesia \\ ${ }^{2}$ Department of Physics, Faculty of Mathematics and Natural Sciences, \\ Padjadjaran University, Indonesia \\ ${ }^{3}$ Division of Electrical, Electronic and Information Engineering, Faculty of Engineering, \\ Osaka University, Japan \\ E-mail: rahmat@fi.itb.ac.id
}

\begin{abstract}
We have studied the preparation of organic-inorganic hybrid polymer precursors by sol-gel technique and their utilization for nanostructured optical components for photonic applications. The gel polymer precursors were prepared from siloxane modified by polymerizable acrylate groups, which can be processed further by photopolymerization process. Molecular structure characterizations by means of the FTIR measurements indicate the conversion of $\mathrm{C}=\mathrm{C}$ bonds into $\mathrm{C}-\mathrm{C}$ bonds after photopolymerization. This bond conversion produces high cross-linking between the organic and inorganic moieties, resulting in thermally stable and chemically resistant thin polymer layer which provide unique advantages of this material for particular optical/photonic applications. By employing laser interference technique, gratings with periodicity between $400-1000 \mathrm{~nm}$ have been successfully fabricated. Application of those sub-micron periodicity of grating structure as active elements in optically pumped polymer laser system and Surface Plasmon Resonance (SPR) based measurement system have been also explored. The experimental results therefore also show the potential applications of this hybrid polymer as a building material for micro/nano-photonics components.
\end{abstract}

Keywords: hybrid polymers; nano-optics; optically pumped polymer laser; pulsed laser interference; sol-gel materials; surface Plasmon resonance.

\section{Introduction}

Functional polymers have attracted much attention for various applications in conventional optics, micro/nano-optical components, such as photonic band gap (PBG) structure, and micro-electro-mechanic systems (MEMS) [1-6].Easiness in fabrication process, which commonly does not require high temperature, is one of the main advantages of polymers. Such processing condition allows the

Received January $11^{\text {th }}, 2011$, Revised February $28^{\text {th }}, 2012$, Accepted for publication May $20^{\text {th }}, 2012$.

Copyright (C) 2012 Published by LPPM ITB \& PII, ISSN: 1978-3051, DOI: 10.5614/itbj.eng.sci.2012.44.3.1 
incorporation of various kinds of functional organic dyes. High temperature requirement in glass and ceramic processing prevents the incorporation of functional organic dyes, which will decompose a thigh temperature. Acrylic and epoxy polymers, such as poly(methylmetacrylate) (PMMA) and SU-8, are typical examples of polymers commonly used for those purposes, including modern optic applications, such as fiber optics, optical interconnect components, solid-state dye lasers, and optical amplifiers [1,2,7-9].However, some disadvantages related to optical transparency, thermal stability, chemical resistance and mechanical strength often restrict further development of their applications for functional optical and photonic components.

In the last decade, organic-inorganic hybrid polymers, which are also called as Ormosils or ORMOCERs polymers, have been much investigated because of their interesting physical-chemical properties [10-14].These hybrid polymers are commonly made from silicon alkoxides that are modified with polymerizable organic groups, such as acrylic, methacrylic or epoxy, resulting in the formation of inorganic and organic cross-linked network. According to the classification given by Sanchez, these kinds of hybrid polymers are classified as Class II, where the organic and inorganic components are linked together by strong chemical bonds (covalent or ionic bonding) [10,11]. The inorganic network is formed by sol-gel process, which is adapted from the solgel method commonly appliedfor preparing sol-gel derived inorganic glasses and Class I organic-inorganic hybrid materials since more than three decades ago. The Class I hybrid materials, which are derived from tetraethoxysilane (TEOS) or tetramethoxysilane (TMOS), have been successfully employed for various applications ranging from bulk glasses to optical fibers, thin film coatings, ultra-pure powders and multifunctional materials [15-17].However, it seems that those Class I hybrid materials are not feasible to be applied for building complicated structures, particularly micro/nano-structures.

In Class II hybrid polymers, the organic network can be formed by photopolymerization of the polymerizable organic groups, offering the possibility of fabrication methods by photolithography, laser direct writing or other novel fabrication techniques [13,14]. Fabrication of micro and nanostructured optical/photonic components using these hybrid polymers have been demonstrated by using those techniques $[13,14,18,19]$. This paper presents the precursor gel preparation of this class of hybrid polymer from methacrylateester modified siloxane by sol-gel technique and its application for constructing diffractive optic based components, such as Distributed Feedback (DFB) grating for photopumped lasers and grating based Surface Plasmon Resonance (SPR) coupler. In this work, laser interference technique using high power pulsed UV laser was applied for fabricating those gratings, which is distinctly different from the fabrication methods in previous reports mentioned above 
$[18,19]$.

\section{Methodology}

Precursor gels of hybrid polymers were synthesized from 3(Trimethoxysilyl)propyl methacrylate (TMSPMA)by sol-gel route. Monomer solution was prepared by dissolving TMSPMA monomer in a mixture solution of water and ethanol. For use in the fabrication of DFB grating for photopumped lasing purpose, this monomer solution was added with organic laser dye, either Rhodamine 6G (R6G) or4-dicyanmethylene-2-methyl-6-(pdimethyl-aminostyryl)-4H-pyran (DCM).However, this monomer solution wasused without addition of any organic laser dye for use in the fabrication of SPR grating coupler. Those monomer solutions were then processed by sol-gel method as described elsewhere with some modification in its processing conditions, until gel formed as its final product [20].Thin films of polymer precursor gel were prepared by spin casting technique after addition of a small amount of photoinitiator (Irgacure 819) into the precursor gel solution. Those gel films were subjected to UV photocuring using a $\mathrm{Hg}$ lamp or a semiconductor laser $(405 \mathrm{~nm})$. Basic molecular structure characterization was carried out by means of FTIR spectroscopy, whereas basic optical characterizations were carried out by UV-Vis spectroscopy and thin film reflectometry.

Fabrication ofDFB grating structure with laser interference technique was performed by using Lloyd mirror configuration, as illustrated schematically in Figure1, and the frequency-tripled (THG) output of Nd-YAG laser(355 nm) as the coherent light source. The interfering light beams at the precursor gel film will be absorbed by photoinitiator resulting in photopolymerization rate that varies spatially following the formed interference pattern. Those laser interfering beams both in scribe interference pattern and cure the precursor gel. Therefore, the wavelength of this laser beam can be also referred as curing wavelength, namely $\lambda_{\text {curing }}=355 \mathrm{~nm}$. The laser power was about $200 \mathrm{~mW}$ with beam diameter of about $1.2 \mathrm{~cm}$, which is equivalent to laser fluence of about 18 $\mathrm{mJ} / \mathrm{cm}^{2}$. The pulse duration was about $10 \mathrm{~ns}$ and the number of pulses was about 2-5times. The grating surface structure was investigated by Atomic Force Microscopy (AFM). The grating periodicities were determined from those AFM images.

In order to investigate the possibility and characteristics of photopumped lasing in those fabricated DFB gratings, the grating was placed in a photopumped laser configuration optically pumped by a strip-line shaped laser beam from the frequency doubled Nd-YAG laser $\left(\lambda_{\text {pump }}=532 \mathrm{~nm}\right.$ ). In order to investigate the fabricated grating as a SPR grating coupler, the grating was previously covered 
by very thin Ag layer of about $50 \mathrm{~nm}$ thickness. This coupler element was then placed and evaluated in a SPR spectroscopy system as described in literatures [21,22].

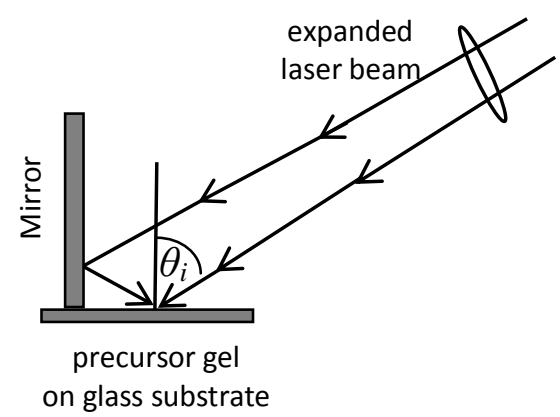

Figure 1 Setup of laser interference using Lloyd mirror for grating structure fabrication. $\theta_{i}$ is the incident angle of laser beam.

\section{Results and Discussion}

\subsection{Molecular Characterizations of Products}

The whole reaction of sol-gel process involves the hydrolysis of silicon alkoxides and then followed by a cascade of condensation and polycondensation reactions, resulting in the formation of inorganic Si-O-Si network, as indicated in the Scheme $1[11,14]$.The photopolymerization reaction occurs through radical polymerization mechanism which involves radical initiation and chain elongations resulting in the formation of cross-linked methacrylate network, as indicated in Scheme 2 [14]. After photopolymerization, according to Scheme 2, there will be a change in molecular structure due to the conversion of carbon-carbon double bond $(\mathrm{C}=\mathrm{C})$ into carbon-carbon single bond $(\mathrm{C}-\mathrm{C})$ in methacrylic group.

Scheme 1. Reactions involved in sol-gel process, where $R$ is methoxy group.

$$
\begin{array}{lll}
\equiv \mathrm{SiOR}+\mathrm{H}_{2} \mathrm{O} & \rightarrow & \equiv \mathrm{SiOH}+\mathrm{ROH} \\
\equiv \mathrm{SiOH}+\mathrm{HO}-\mathrm{Si} \equiv & \rightarrow & \equiv \mathrm{Si}-\mathrm{O}-\mathrm{Si} \equiv+\mathrm{H}_{2} \mathrm{O} \\
\equiv \mathrm{SiOH}+\mathrm{RO}-\mathrm{Si} \equiv & \rightarrow & \equiv \mathrm{Si}-\mathrm{O}-\mathrm{Si} \equiv+\mathrm{ROH}
\end{array}
$$

Scheme 2. Reactions involved in photopolymerization, where $I$ is initiator molecule, $r^{*}$ is radical and $R^{\prime}$ is methacrylic group.

$$
\mathrm{I}+k T\left(\text { or } h v_{1}\right) \quad \rightarrow \quad 2 \mathrm{r}^{*}
$$




$$
\begin{array}{ll}
\mathrm{r}^{*}+\equiv \mathrm{SiR}^{\prime} \quad \rightarrow \quad \mathrm{r}\left(\equiv \mathrm{SiR}^{\prime}\right)^{*} \\
\mathrm{r}\left(\equiv \mathrm{SiR}^{\prime}\right)_{n}{ }^{*}+\mathrm{r}\left(\equiv \mathrm{SiCR}^{\prime}\right)_{m}{ }^{*} & \rightarrow 2\left(\mathrm{r}\left(\equiv \mathrm{SiR}^{\prime}\right)_{n+m}\right)
\end{array}
$$

Figure 2 shows the FTIR spectra of hybrid polymer precursor gel measured before and after photopolymerization. In comparison between the FTIR spectrum of TMSPMA monomer and the precursor gel (before photopolymerization), infrared absorption bands at $v=\sim 800 \mathrm{~cm}^{-1}$ and $1100 \mathrm{~cm}^{-1}$ were significantly suppressed [23].Those absorption bands are related to the suppression of symmetric and asymmetric stretching of $\mathrm{Si}-\mathrm{O}$ bonds, respectively. The suppression of those bands thus indicates the formation of inorganic network. As evident in Figure2, after photopolymerization, the absorption band at $1635 \mathrm{~cm}^{-1}$ decreases indicating the reduction of $\mathrm{C}=\mathrm{C}$ vibration due to the conversion as mentioned above. A broad absorption band in the region of $3500 \mathrm{~cm}^{-1}$ is assigned to $\mathrm{O}-\mathrm{H}$ vibration, which may be due to the presence of the remaining water, ethanol and unreacted $\mathrm{O}-\mathrm{H}$ group inside the precursor gel. Water and ethanol are expected to evaporate or disappear from the film during post-baking process after photopolymerization step, but the unreacted hydroxyl $(\mathrm{O}-\mathrm{H})$ groups may be still remain present in the film. This $\mathrm{O}-\mathrm{H}$ vibration band therefore becomes smaller but still remains in the FTIR spectrum taken after photopolymerization in methacrylic group.

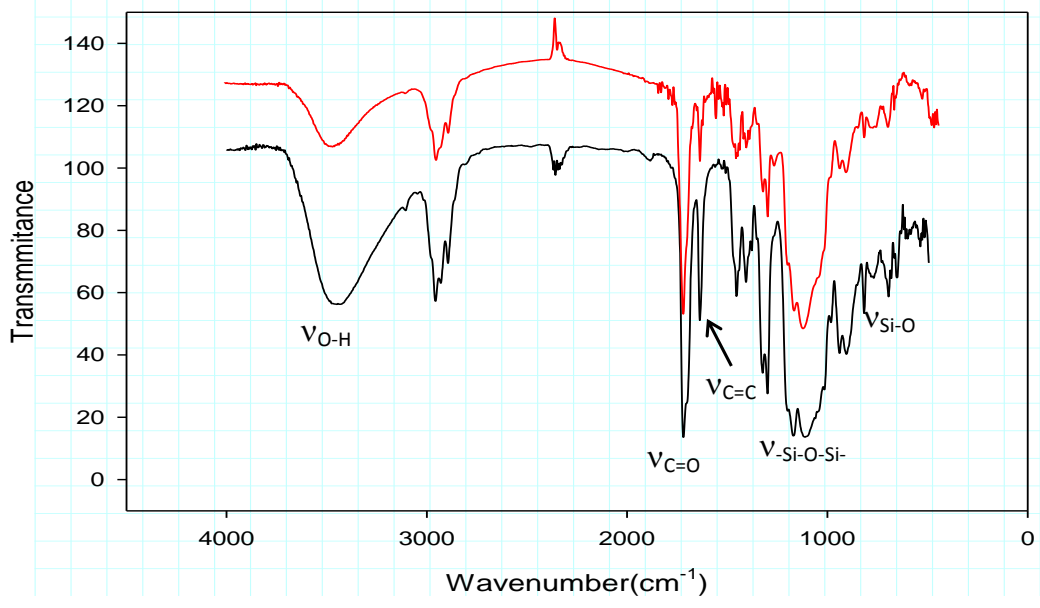

Figure 2 FTIR spectra of precursor gel after photopolymerization (red line, upper side spectrum) and precursor before photopolymerization (black line, lower side spectrum).

\subsection{Grating Fabrication and Characterizations}

Grating fabrication was performed in laser interference setup, as described in 
the methodology section, at certain incident angle $\left(\theta_{i}\right)$ of interfering laser beams with respect to the normal of the precursor gel film surface. The dependence of grating periodicity $(\Lambda)$ on incident angle and curing wavelength (the interfering laser beams wavelength)is given by:

$$
\Lambda=\frac{\lambda_{\text {curing }}}{2 \sin \left(\theta_{i}\right)}
$$

Using that formula, the grating periodicities were estimated to be about 1022, $454,420,391$, and $366 \mathrm{~nm}$ for incident angles of $10^{\circ}, 23^{\circ}, 25^{\circ}, 27^{\circ}$, and $29^{\circ}$, respectively. Figure 3(a) and 3(b) shows the AFM images of a grating structure prepared at the incident angle of interference laser of $10^{\circ}$. This grating has periodicity of about $1000 \mathrm{~nm}$, which is close to the estimated value above.

As depicted from the AFM images, sinusoidal corrugation structure was formed with the grating depth of about 20-45 nm. The formation mechanism of this corrugated grating structure is still not well understood at this stage, however we suppose the formation may occur through some possible mechanisms as followings. The first possibility may be related with polymerization induced volume shrinkage. At the bright region, high laser intensity produces high degree of photopolymerization which causes volume shrinkage as the precursor gel changing into solid. On the other hand, at the dark region, photopolymerization occurs with relatively low reaction rate so that there is no significant volume shrinkage. As the bright region suffers more volume shrinkage, the bright region would create valley while the dark region will create bump. However, there is also other possible formation mechanism that includes monomer diffusion during the polymerization. As the monomer concentration quickly vanishes at the bright region due to high polymerization rate, this condition leads to the diffusion of monomer from dark region to bright region. The monomers are then attracted and accumulated at the bright region. This mechanism, which is called as photo-induced swelling, results in bump formation at the bright region but valley formation at the dark region $[18,19]$.

It should be also noted that, however, in this work the grating structure was formed by using pulse laser energy of about $20 \mathrm{~mJ} /$ pulse, which is equivalent to light power of about $2 \mathrm{MW} / \mathrm{cm}^{2}$ per pulse. This fabrication condition is significantly different from that found in the fabrication method used in the previous reports $[18,19]$. A light beam with such optical power may theoretically generate optical radiation force in the order of $10^{-3} \mathrm{~N} / \mathrm{cm}^{2}$ [24,25]. Regarding to this fact, though it is still speculative at the moment, one may also suggest the mechanism formation due to radiation pressure effect. When the interfering laser beams with high laser impinge on the surface of the precursor gel film, the light momentum will be transferred to the precursor molecules. If the precursor 
gels is considered to be composed of nano-sizeparticulates, [14] this momentum transfer may lead to the displacement of those precursor gel particulates in the same direction with the laser beam propagation direction. However, in the same time, the laser beam also induces cross linking photopolymerization and prevent the precursor to move back. This process creates bump at the dark region and valley at the bright region, producing a corrugation structure mimicking the intensity profile of interfering laser beams. Such process will not happen in SU8 resin because the radiation force is not sufficiently enough to displace these resin molecules which have much larger molecular weight. Further specific experimental works, however, are required to verify which one of those possible mechanisms explained above actually responsible for the grating formation here is.
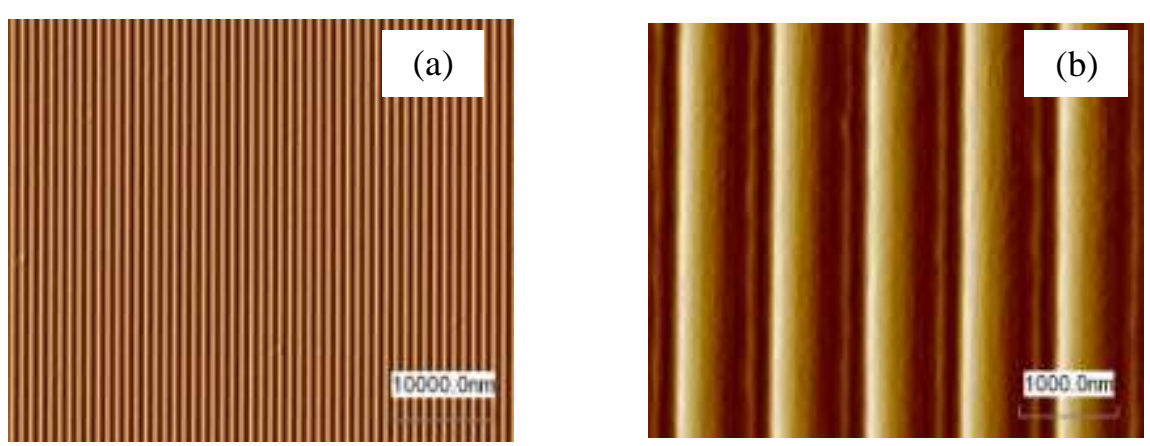

Figure 3 (a) Atomic Force Microscopy (AFM) images of a grating fabricated at incident angle of laser interference beam of $10^{\circ}$. (b) The same image sample but taken in smaller scale.

\subsection{Application as Distributed Feedback Grating and Photo- pumped Lasing}

Fabricated DBF grating evaluated in a photopumped laser configuration, which is optically pumped by the frequency doubled Nd-YAG pulse laser. Figure 4(a) shows the emission spectra measured from hybrid polymer DFB grating containing $\mathrm{R} 6 \mathrm{G}$ organic laser dye. At low laser pumping energy, only fluorescence spectrum was observed from the sample. A thigh laser pumping energy, the emission spectral shape then changes indicated by much narrower spectral width with FWHM less than $2 \mathrm{~nm}$, which is in the limit range of the spectrophotometer resolution $(\sim 1 \mathrm{~nm})$. Figure 4 (b) shows that the emission spectral width and peak intensity start to change abruptly at the pumping laser intensity of about $0.1 \mathrm{~mJ} / \mathrm{pulse}$, which is the threshold pumping energy. This clearly indicates the generation of lasing actions in this fabricated DFB grating structure. The relationship between lasing wavelength, Bragg wavelength and grating periodicity is given by 


$$
\lambda_{\text {lasing }}=\frac{\lambda_{\text {Bragg }}}{m}=\frac{2 n_{\text {eff }} \Lambda}{m}
$$

where $m$ is the Bragg reflection order, and $n_{\text {eff }}$ is the effective refractive index. As evident in Figure 4(a), lasing was observed at about $580 \mathrm{~nm}$ from a grating prepared at incident angle of $27^{\circ}$. The observed lasing wavelength is in agreement with the estimated lasing wavelength calculated using Eq.(2), for $m=$ 2 , as indicated in Table 1 . This fact also indicates that the lasing occurs at the second order $(m=2)$ of Bragg reflection wavelength.
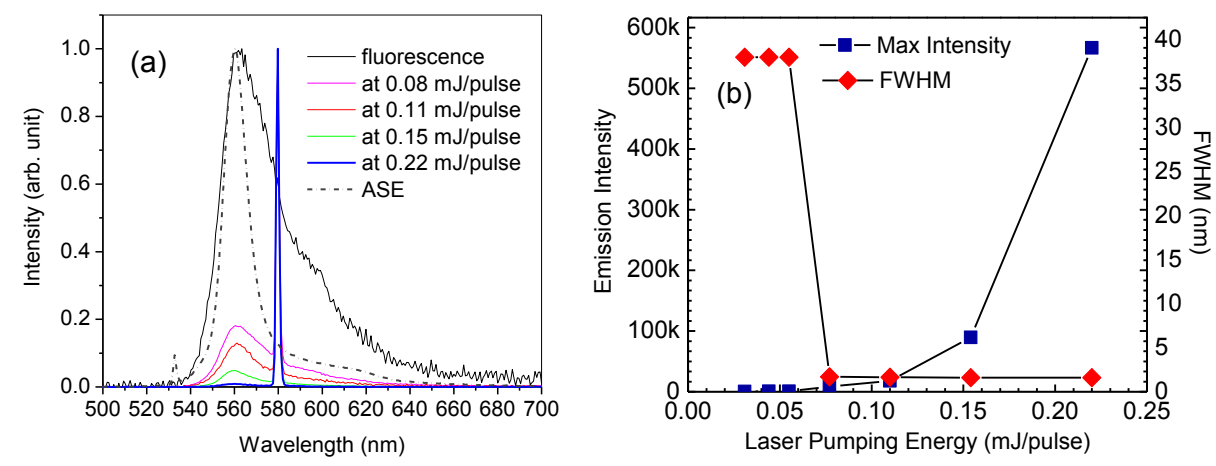

Figure 4(a) The observation of lasing action in a hybrid polymer grating containingR6G laser dye, which is indicated by the narrowing of its emission spectral width. Fluorescence and Amplified Spontaneous Emission (ASE) spectra are also displayed for comparison. (b) The plots of emission peak intensity and emission spectral width depending on the laser pumping energy.

It should be noted that the lasing performance, indicated by lasing wavelength and threshold energy, is critically dependent on the geometrical structure of the grating, such as the grating periodicity and corrugation depth. As the grating formation may involve volume shrinkage or photo-induced swelling mechanism, the deviation in geometrical structure may occur at each time of fabrication leading to unpredictable degree of deviation in its performance. It is therefore necessary to study further in separate work how to improve the precursor gel and the fabrication technique in order to minimize geometrical structure deviation. 
Table 1 The estimated grating periodicities and lasing wavelengths $v s$. incident angles used in the grating fabrication (calculated with the assumption $n=1.5$ ).

\begin{tabular}{ccc}
\hline $\begin{array}{c}\text { Incident } \\
\text { Angle }\end{array}$ & $\begin{array}{c}\text { Estimated } \\
\text { Periodicity (nm) }\end{array}$ & $\begin{array}{c}\text { Estimated Lasing Wavelength } \\
\text { (at the } 2^{\text {nd }} \text { order Bragg wavelength) } \\
(\mathbf{n m})\end{array}$ \\
\hline 10 & 1022 & 1533 \\
23 & 454 & 681 \\
25 & 420 & 630 \\
27 & 391 & 586 \\
\hline
\end{tabular}

\subsection{Application as Coupling Elements for Surface Plasmon Resonance Generation}

Surface Plasmon Resonance (SPR) is a collective oscillation of electrons at the flat metal surface (at the metal/dielectric interface), which is in resonant condition with the incoming electromagnetic or light wave. The condition for resonance is given by $[13,14]$ :

$$
K_{s p}=k_{o} \sqrt{\frac{\sqrt{\varepsilon_{1} \varepsilon_{2}}}{F_{1}+\varepsilon_{2}}}
$$

where $K_{s p}$ is the propagation constant of the SPR wave, while $\varepsilon_{1}$ and $\varepsilon_{2}$ are the permittivity of metal and dielectric, respectively. In this case, surface wave can be generated by using a prism coupler, which is constructed of a flat metal coated on a prism. In the present work, we fabricated SPR grating couplers that are constructed from a hybrid polymer grating covered by a very thin metal layer (approx. $50 \mathrm{~nm}$ ) on its top surface. Such SPR grating coupler has attractive much attention for various bio-chemical molecular sensing applications [26-28]. The resonance condition for grating coupled SPR configuration is:

$$
K_{S P}=K_{i}+m \frac{2 \pi}{\Lambda}
$$

where $K_{i}$ is the propagation constant of the incoming light along the grating surface, $\Lambda$ is the grating periodicity and $m$ is an integer $(= \pm 1, \pm 2, \ldots)$ that denotes the diffraction order [26]. 

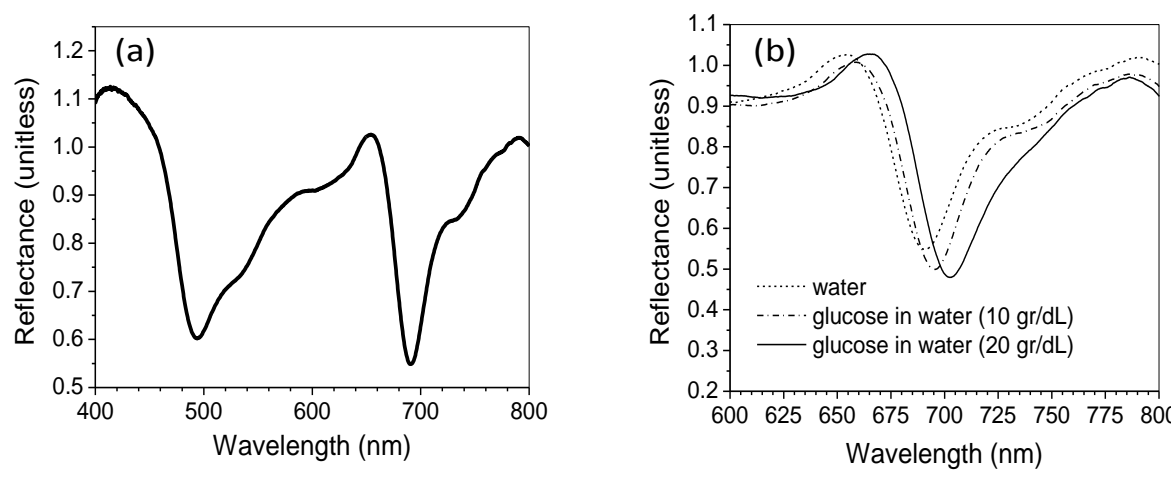

Figure 5 (a) The spectrum of Surface Plasmon Resonance (SPR) measured by using SPR grating coupler prepared in this work from hybrid polymer precursor gel. (b) The shifts of the SPR dip depending on the refractive index of dielectric solution which is in contact with this SPR grating coupler element.

The fabricated grating, from precursor gel without addition of any laser dye substance, was covered by thin film silver layer with about only $50 \mathrm{~nm}$ in thickness. The grating periodicity is about $670 \mathrm{~nm}$. Figure 5(a) shows the SPR spectrum measured for water as the dielectric layer, which is in direct contact with the silver layer. The spectrum shows two dips, which is typical SPR spectrum obtained by using grating coupled SPR configuration. This is distinctly different from SPR spectrum measured from SPR system with prism coupled configuration, which normally exhibits only one spectrum dip. In the case of grating coupler SPR element, the Plasmon wave suffers Bragg reflection leading to the formation of standing wave. Such condition creates a forbidden gap and splits the dispersion curve at the cross-section, as commonly observed in photonic crystal theory [29]. The SPR spectrum now therefore exhibits two SPR dips. The spectral shape of the dip is much narrower and deeper in comparison to that of measured by using just flat metal as in prism-coupled SPR system. Figure 5(b) the shifting of SPR dip from $691 \mathrm{~nm}$ to $695 \mathrm{~nm}$ and $702 \mathrm{~nm}$ with increasing glucose concentration in water from $0 \mathrm{~g} / \mathrm{dL}$ to $10 \mathrm{~g} / \mathrm{dl}$ and 20 $\mathrm{g} / \mathrm{dL}$, respectively. This concentration change is corresponding to the change of solution refractive index from 1.30 to 1.32 and 1.34, respectively. This result demonstrates that the fabricated grating performs well as a SPR grating coupler, which can be applied in the refractive index measurement applications. 


\section{Conclusion}

Precursor gels of hybrid polymers containing polymerizable methacrylate group have been prepared via sol-gel route. Grating structure has been successfully fabricated on the surface of this hybrid polymer film by laser interference technique utilizing high power UV pulse laser. The resulted gratings have sinusoidal corrugated structure with periodicity in the range $400-1000 \mathrm{~nm}$, which can be selected by adjusting the incident angle. The mechanism formation still cannot clearly verified at this stage, but it may involve an interesting mechanism by considering the time duration required for the grating formation. The applications of those fabricated grating structures for generation of photopumped lasing and SPR wave have been also successfully demonstrated. The present experimental results thus also demonstrate the feasibility of this hybrid polymer as a building material for nanostructured optical/photonic components.

\section{Acknowledgments}

The authors would like thank to JSPS (Japan) and DGHE (Indonesia) for the research support under JSPS/DGHE Joint Research Project 2007-2010 scheme. Part of this work was supported by Program Riset Kelompok Keahlian ITB 2010 (contract no. 234/K01.7/PL/2010).

\section{References}

[1] Kuzyk, Mark G., Polymer Fiber Optics: Materials, Physics, and Applications (Optical Science and Engineering; 117), CRC Press, Taylor \& Francis Group, New York, 2007.

[2] Kuriki, K., Koike, Y. \& Okamoto, Y., Plastic Optical Fiber Lasers and Amplifiers Containing Lanthanide Complexes, Chem. Rev., 102, pp. 2347-2356, 2002.

[3] Edrington, A.C., Urbas, A.M., DeRege, P., Chen, C.X., Swager, T.M., Hadjichristidis, N., Xenidou, M., Fetters, L.J., Joannopoulos, J.D., Fink, Y. \& Thomas, E.L., Adv. Mater., 13, Polymer-Based Photonic Crystals, pp. 421-425, 2001.

[4] Psaltis, D., Quake, S.R. \& Yang, C., Developing Optofluidic Technology Through The Fusion of Microfluidics and Optics, Nature, 442, pp. 381386, 2006.(doi:10.1038/nature05060)

[5] Leeds, A.R., Van Keuren, E.R., Durst, M.E., Schneider, T.W., Currie, J.F. \& Paranjape, M., Integration of Microfluidic and Microoptical Elements Using A Single-Mask Photolithographic Step,Sensors and Actuators A, 115, pp. 571-580, 2004.

[6] Biswas, A., Friend, C.S. \& Prasad, P.N., Encyclopedia of Materials: Science and Technology, Elsevier Science Ltd., 2000. 
[7] Sorek, Y. \& Reisfeld, R., Sol-Gel Glass Wave Guides Prepared at Low Temperature, Appl. Phys. Lett., 63, p. 3256, 1993.

[8] Kobayashi, T., Nakatsuka, S., Iwafuji, T., Kuriki, K., Imai, N., Nakamoto, T., Claude, C.D., Sasaki, K., Koike, Y. \& Okamoto, Y., Fabrication and Superfluorescence of Rare-Earth Chelate-Doped Graded Index Polymer Optical Fibers,Appl. Phys. Lett. 71,p. 2421, 1997; Slooff, L.H., van Blaaderen, A., Polman, A., Hebbink, G.A., Klink, S.I., van Veggel, F.C.J.M., Reinhoudt, D.N., \& Hofstraat, J.W., Rare-earth Doped Polymers for Planar Optical Amplifiers, J. Appl. Phys., 91, p. 3955, 2002.

[9] Hidayat, R., Sugihara, O.,Tsuchimori, M., Kagami, M., Nishikubo, T. \& Kaino, T., Binding of Europium Complex to Polymerizable Macrocyclic Molecules and its Optical Properties, Opt. Mat, 29, p. 1367-1374, 2007.

[10] Sanchez, C., Julian, B., Belleville, P. \& Popall, M., Applications of Hybrid Organic-Inorganic Nanocomposites, J. Mater. Chem., 15, pp. 3559-3592, 2005.

[11] Novak, B.M., Hybrid Nanocomposite Materials between Inorganic Glasses and Organic Polymers, Adv. Mater., 5, pp. 422-433, 1993.

[12] Schottner, G., Hybrid Sol-Gel-Derived Polymers: Applications of Multifunctional Materials, Chem. Mater., 13, pp. 3422-3435, 2001.

[13] Buestrich, R., Kahlenberg, F., Popall, M., Dannberg, P., Muller-Fiedler, R. \& Rosch, O., ORMOCER ${ }^{\circledR}$ for Optical Interconnect Technology, J. Sol-Gel Sci. Technol.,20, pp. 181-186, 2001.

[14] Haas, K. \& Wolter, H., Hybrid Inorganic/Organic Polymers with Nanoscale Building Blocks: Precursors, Processing, Properties and Applications, Rev. Adv. Mater. Sci., 5, pp. 47-52, 2003.

[15] Wen, J. \& Wilkes, G.L., Organic/Inorganic Hybrid Network Materials by The Sol-Gel Approach, Chem. Mater., 8, pp. 1667-1681, 1996.

[16] Kirkbir, F., Murata, H., Mayer, D., Chaudhari, S.R., \& Sarkar, A., Drying and Sintering of Sol-Gel Derived Large $\mathrm{SiO}_{2}$ Monoliths, J. Sol-Gel Sci. Technol.,6,pp. 203-217,1996; Nogues, J.L.R. \& Moreshead, W.V., Porous Gel-Silica, A Matrix for Optically-Active Components, J. NonCryst. Solids., 121, pp. 136-142,1990.

[17] Burzynski, R. \& Prasad, P.N., Photonics and Nonlinear Optics with SolGel Processed Inorganic Glass: Organic Polymer Composite, Klein L.C. (ed.), Kluwer, Boston, Chapter 19.

[18] Croutxé-Barghorn, C., Soppera, O. \& Chevallier, M., Diffraction Gratings in Hybrid Sol-Gel Films: on The Understanding of The Relief Generation Process, Macromol. Mater. Eng., 288, pp. 219-227, 2003.

[19] Blanc, D., Pélissier, S., Jurine, P.Y., Soppera, O., Croutxé-Barghorn, C. \& Carré, C., Photo-Induced Swelling of Hybrid Sol-Gel Thin Films: Application to Surface Micro-Patterning, J. Sol-Gel Sci. Tech., 27, pp. 215-220,2003. 
[20] Fan, X., Wua, X., Wanga, M., Qiub, J. \& Kawamoto, Y., Luminescence Behaviors of $\mathrm{Eu}^{3+} \beta$-Diketonate Complexes in Sol-Gel-Derived Host Materials, Mater. Lett., 58, p. 2217,2004; Fan, X., Lia, W., Wang \& F. Wang, M., Luminescence Behavior of the Europium (III) Complexes with Hexafluoracetylacetonate in the ORMOSIL Matrices, Mat. Sci. \& Eng. B, 100, p. 147, 2003.

[21] Homola, J., Surface Plasmon Resonance Based Sensors, Springer: New York, 2006.

[22] Maier, S.A., Plasmonics: Fundamentals and Applications, Springer: United Kingdom, 2007.

[23] http://www.sigmaaldrich.com/spectra/ftir/FTIR003510.PDF (accessed in Sept. $3^{\text {rd }}, 2010$ )

[24] Loudon, R., Theory of the Radiation Pressure on Dielectric Surfaces, J. Mod. Opt., 49, pp. 821-838, 2002.

[25] Mansuripur, M., Radiation Pressure and the Linear Momentum of the Electromagnetic Field, Opt. Expr., 12, p. 5375,2004.

[26] Homola, J., Surface Plasmon Resonance Sensors for Detection of Chemical and Biological Species, Chem. Rev., 108, pp. 462-493, 2008; Dostalek, J. \& Homola, J., Surface Plasmon Resonance Sensor Based on an Array of Diffraction Gratings for Highly-Parallelized Observation of Biomolecular Interactions, Sens. and Act. B, 129, pp. 303-310, 2008.

[27] Roh, S., Chung, T. \& Lee, B., Overview of the Characteristics of Microand Nano-Structured Surface Plasmon Resonance Sensor, Sensors, 11, pp. 1565-1588, 2011.

[28] Yu, F., Tian, S., Yao, D. \& Knoll, W., Surface Plasmon Enhanced Diffraction for Label-Free Biosensing, Anal. Chem., 76, pp. 3530-3535, 2004.

[29] Sakoda, K., Optical Properties of Photonic Crystal, Springer: Berlin, 2004. 